\title{
A human factors intervention in a hospital - evaluating the outcome of a TeamSTEPPS program in a surgical ward
}

\author{
Oddveig Reiersdal Aaberg ${ }^{1,2,3^{*}}$ (D), Marie Louise Hall-Lord ${ }^{2,4}$ (D), Sissel Iren Eikeland Huseb $\varnothing^{3,5}$ (D) and
} Randi Ballangrud² (D)

\begin{abstract}
Background: Patient safety in hospitals is being jeopardized, since too many patients experience adverse events. Most of these adverse events arise from human factors, such as inefficient teamwork and communication failures, and the incidence of adverse events is greatest in the surgical area. Previous research has shown the effect of team training on patient safety culture and on different areas of teamwork. Limited research has investigated teamwork in surgical wards. The aim of this study was to evaluate the professional and organizational outcomes of a team training intervention among healthcare professionals in a surgical ward after 6 and 12 months. Systems Engineering Initiative for Patient Safety 2.0 was used as a conceptual framework for the study.
\end{abstract}

Methods: This study had a pre-post design with measurements at baseline and after 6 and 12 months of intervention. The intervention was conducted in a urology and gastrointestinal surgery ward in Norway, and the study site was selected based on convenience and the leaders' willingness to participate in the project. Survey data from healthcare professionals were used to evaluate the intervention. The organizational outcomes were measured by the unit-based sections of the Hospital Survey of Patient Safety Culture Questionnaire, and professional outcomes were measured by the TeamSTEPPS Teamwork Perceptions Questionnaire and the Collaboration and Satisfaction about Care Decisions in Teams Questionnaire. A paired t-test, a Wilcoxon signed-rank test, a generalized linear mixed model and linear regression analysis were used to analyze the data.

Results: After 6 months, improvements were found in organizational outcomes in two patient safety dimensions. After 12 months, improvements were found in both organizational and professional outcomes, and these improvements occurred in three patient safety culture dimensions and in three teamwork dimensions. Furthermore, the results showed that one of the significant improved teamwork dimensions "Mutual Support" was associated with the Patient Safety Grade, after 12 months of intervention.

(Continued on next page)

\footnotetext{
* Correspondence: oddveig.aaberg@ntnu.no

'Department of Health and Nursing Science, Faculty of Health and Sport Sciences, University of Agder, Universitetsveien 25 A, 4630 Kristiansand, Norge

${ }^{2}$ Department of Health Science, Faculty of Medicine and Health Sciences, Norwegian University of Science and Technology, Teknologivegen 22, 2815 Gjøvik, Norway

Full list of author information is available at the end of the article
}

(c) The Author(s). 2021 Open Access This article is licensed under a Creative Commons Attribution 4.0 International License, which permits use, sharing, adaptation, distribution and reproduction in any medium or format, as long as you give appropriate credit to the original author(s) and the source, provide a link to the Creative Commons licence, and indicate if changes were made. The images or other third party material in this article are included in the article's Creative Commons licence, unless indicated otherwise in a credit line to the material. If material is not included in the article's Creative Commons licence and your intended use is not permitted by statutory regulation or exceeds the permitted use, you will need to obtain permission directly from the copyright holder. To view a copy of this licence, visit http://creativecommons.org/licenses/by/4.0/ The Creative Commons Public Domain Dedication waiver (http://creativecommons.org/publicdomain/zero/1.0/) applies to the data made available in this article, unless otherwise stated in a credit line to the data. 
(Continued from previous page)

Conclusion: These results demonstrate that the team training program had effect after 12 months of intervention. Future studies with larger sample sizes and stronger study designs are necessary to examine the causal effect of a team training intervention in this context.

Trial registration number: ISRCTN13997367 (retrospectively registered).

Keywords: Human factors, Implementation, Intervention, Interprofessional teamwork, Longitudinal, Patient safety culture, SEIPS 2.0, TeamSTEPPS, Team training

\section{Background}

Patient safety in hospitals is being jeopardized, since too many patients experience adverse events $[1,2]$. The risk of adverse events in surgical care is higher than in other areas of hospitals [3, 4]. Most adverse events arise not from the solitary actions of individuals but from the systems of which they are a part and with which they interact [5]. Root cause analyses have revealed that human factors, such as poor teamwork and communication failures, are the underlying factors for the majority of adverse events in hospitals [2, 6]. Focusing on patient safety culture is crucial for minimizing adverse events and improving patient safety [7]. An organization's patient safety culture is the product of individual and group values, beliefs, attitudes, perceptions, competencies, and patterns of behavior that determine the organization's commitment to quality and patient safety [8]. Patient safety requires that healthcare professionals have the right competencies and tools to perform their tasks. It is therefore crucial to conduct patient safety interventions that focus on healthcare professionals and work system factors that contribute to safe care [9]. In this study, we conducted a team training intervention in a surgical ward.

The surgical ward is a microsystem within a hospital organization and a unit with a high degree of complexity [10]. The interdependency among healthcare professionals contributes to this complexity [1]. Clinical work requires a broad spectrum of competencies, and healthcare professionals are often working under high time pressure [11]. Surgical ward physicians are often called to the operating room for surgical procedures during a work shift [12], and this makes interprofessional teamwork in the wards extra challenging.

Human factors is a multidisciplinary science at the intersection of psychology and engineering [13] and is commonly described as a discipline devoted to studying and improving the interactions among humans and other elements of a system [14]. Human factors interventions aim to improve system performance and prevent accidental harm, which for healthcare means supporting the cognitive and physical work of healthcare professionals and promoting high-quality, safe care for patients [15]. Human factors interventions, such as team training, are regarded as an innovative approach for improving patient safety [16-18]. Team training is described as applying a set of instructional strategies that rely on well-tested tools (e.g., simulation, lectures, and videos) to achieve specific team competencies $[19,20]$.

Previous research on team training interventions has shown improvements in different areas of teamwork $[21,22]$ and safety culture $[23,24]$, reductions in surgical harm [25], and reductions in surgical mortality [26]. However, most of the team training research has been conducted in specialty units, and limited research has investigated teamwork in surgical wards [27] or investigated teamwork over long time frames [28]. Few studies have examined the associations between perceptions of teamwork and patient safety culture after a 12-month team training intervention. Observational studies have found that interprofessional teamwork was associated with organizational culture [29] and that event reporting, communication, and leadership were predictors of patient safety culture [30].

In this study, we implemented Team Strategies and Tools to Enhance Performance and Patient Safety (TeamSTEPPS ${ }^{\oplus}$ ) in a surgical ward. TeamSTEPPS is a generic program based on research $[31,32]$ and is built on five key principles: "Team Structure" and the four team competencies "Leadership", "Situation monitoring", "Mutual support" and "Communication" [32]. The four team competencies of TeamSTEPPS have 15 associated tools and strategies that are meant to be implemented in clinical practice to improve performance and patient safety [33]. "Team decision making" is an additional team competency or team process $[2,34,35]$ that is not included in the TeamSTEPPS program but was included in this study since it is an important aspect of teamwork and has significance for patient safety and patient care $[34,36]$. Research from other areas of hospitals shows that most clinical decisions are still made independently by medical professionals, with only some sharing of information, and that such decisions are rarely made collectively by the interprofessional care team [37].

Since the need to implement team training programs in the surgical ward context is being increasingly 
recognized, an interprofessional TeamSTEPPS intervention was initiated in a surgical ward. We anticipated that training and implementation of teamwork tools and strategies in daily practice among healthcare professionals would improve professional outcomes in terms of perceptions of teamwork, and organizational outcomes in terms of patient safety culture, since the TeamSTEPPS program focuses on both teamwork and patient safety [32]. It takes time to achieve culture change and to embed and sustain new ways of working. Changes that occur in a short time, due to training experience and excitement, may disappear [23]. Therefore, we measured the effect of the intervention 6 and 12 months after initiation.

The aim of the study was to evaluate the professional and organizational outcomes of a team training intervention among healthcare professionals in a surgical ward after 6 and 12 months. The research questions were as follows:

1. Did professional outcome measured by healthcare professionals' perceptions of teamwork and organizational outcome measured by patient safety culture improve from baseline to 6 and 12 months of intervention?

2. Did patient safety culture related to the intervention vary by profession group or time, demonstrating an effect of the intervention?

3. Were perceptions of teamwork dimensions associated with patient safety culture in the unit after 12 months?

\section{Conceptual framework}

Teamwork and patient safety may be explained on the basis of an input-process-output (IPO) framework that describes the impact of input on process and output, as in classic system theory $[20,34,38]$. The human factors model "The Systems Engineering Initiative for Patient Safety 2.0" (SEIPS 2.0) is an IPO model developed for innovative patient safety research in healthcare [5, 39]. The model emphasizes structural elements in the work system with a person at the center. The person may be represented by patients, healthcare professionals, or healthcare teams - as in this study. The team members perform a range of tasks using various tools and technologies in an internal and external environment and under specific organizational conditions, which all influence the care processes and which in turn influence the outcomes [5, 39]. Unlike most of the IPO models, the SEIPS model differentiates the outcomes in 1) patient outcomes, 2) professional outcomes and 3) organizational outcomes [39]. The interrelatedness of the elements (person, tasks, tools and technology, organization, internal and external environment) within the work system, and among the work system, process and outcome illustrates the complexity of the system [39].

In this study, we used the SEIPS 2.0 model to conceptualize the intervention and the outcomes of the study from a system perspective [40]. Implementation of a team training program was regarded as an input in the organization element to strengthen the work system by attempting to improve healthcare professionals' team competencies and patient safety culture [20,38]. The SEIPS 2.0 model illustrates how input, in the work system, such as team training, may improve healthcare professionals' team competencies and influence work processes that in turn influence professional and organizational outcomes. See Fig. 1.

\section{Methods}

Study design

We conducted a study with a pre-post design with measurements at baseline, after 6 months and after 12 months of intervention.

\section{Setting and sample}

The intervention was conducted in a 20-bed urology and gastrointestinal surgery ward in a 180-bed hospital in Norway. The study site was selected by convenience and based on the leaders' willingness to participate in the project, motivated by patient safety incidents in the ward. The profile of the surgical ward is displayed in Table 1. No major changes in the unit profile occurred during the study period, except for changes in leadership positions (which is specified in the text in the intervention section). All of the 43 frontline healthcare professionals (12 physicians, 24 registered nurses, and 7 nursing assistants) were invited to participate in the study. A total of 41 participated in the 6-h initial team training. Normal turnover among nurse staff and physicians caused changes in the sample size.

\section{The intervention}

The intervention was conducted according to the TeamSTEPPS implementation plan, which comprises three phases, that are based on Kotters change model [32] and aligns with the Clinical Human Factors Group recommendation for team training interventions [41].

\section{Phase 1. Set the stage and decide what to do - assessment and planning}

A site assessment was conducted and an overview of TeamSTEPPS was provided to the leadership of the surgical department and the leaders of the selected ward. After the leaders had decided that their unit was ready for the TeamSTEPPS program, an intervention plan was developed jointly by a project group consisting of the researchers and the leaders of the ward. The leaders consisted of the chair 


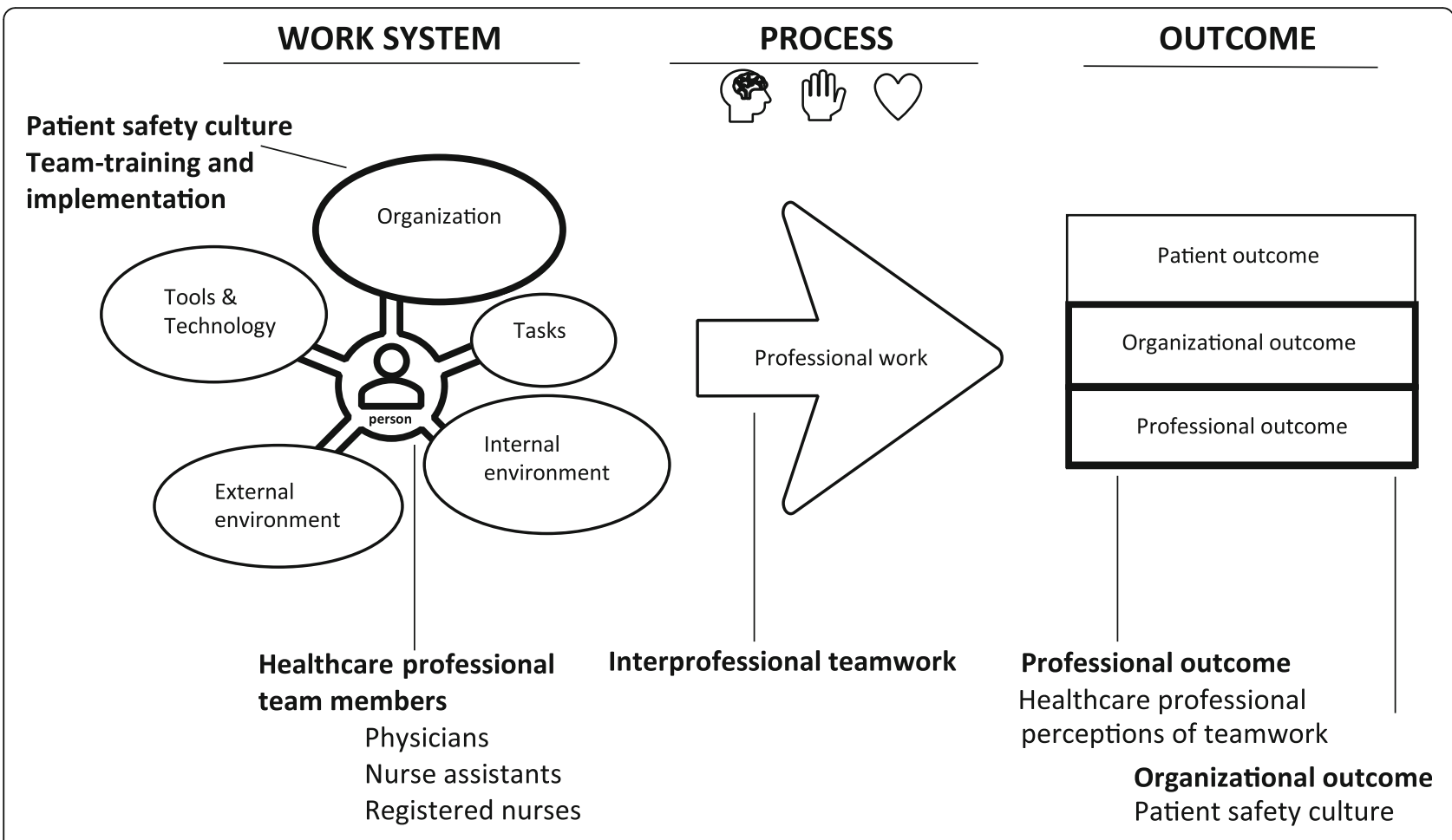

Fig. 1 A modified SEIPS 2.0 model adapted from Holden et al. [39]. The components with the bold lines illustrate the input and outcome in this study from a human factors system perspective

of the surgical department, the unit nurse manager, and the two head surgeons (urology and gastrointestinal surgery). In advance of the intervention start, the physicians and nursing staff attended information meetings conducted by the researchers.

\section{Phase 2. Make it happen - training, planning and implementation}

The onset of the intervention was a mandatory 6-hour interprofessional TeamSTEPPS training distributed over 3 days in a period of 3 weeks. In advance of the training, TeamSTEPPS leaflets and pocket-guides were distributed to all healthcare personnel, which they were asked to read in preparation for the training. The training was conducted in a simulation center at a university and delivered by the master trained nurses and physician leaders in the surgical ward. The team training was a combination of didactics, videos, role play and high-fidelity simulation training. The simulation training included debriefing sessions with a focus on interprofessional teamwork. The first lecture, held by the chair of the surgical department, aimed to create a sense of urgency by presenting the hospital's reports of adverse events. At the end of the training, the healthcare professionals were asked to identify patient safety issues in the ward and to suggest TeamSTEPPS tools to solve the problems. Immediately after the training, the participants responded to the "The TeamSTEPPS
Course Evaluation Survey". The evaluation results were very good, both regarding training satisfaction and learning outcomes [42].

After the training, an interprofessional change team was established. The change team consisted of $12 \mathrm{mem}$ bers representing all levels in the organization, including a former patient and one of the researchers (ORA), and it was led by the unit nurse manager. The researcher coached the change team. Based on the identified safety issues, the change team developed an action plan, according to which they implemented tools and strategies into daily practice. The vision of the action plan was "Zero errors", and the specific goals were aligned with the organizational goals of the surgical department. The unit nurse manager, the clinical nurse specialist, and the two head surgeons, led the implementation in collaboration with the other members of the change team.

Five tools were implemented in the ward during the first 6 months of the study period, at a rate of approximately one tool per month (Table 3). The tool of the month was communicated through weekly newsletters and staff meetings and implemented in daily practice. A description of the selected tools and strategies implemented in the ward is displayed in Table 2, and an overview of the start times of a new tool to be implemented is displayed in Table 3. Refresher training for the nursing 
Table 1 Unit profile data

\begin{tabular}{|c|c|c|c|}
\hline & Baseline & 6 months & 12 months \\
\hline \multicolumn{4}{|l|}{ Beds and nurse/bed ratio } \\
\hline Number of patient beds & 20 & 20 & 20 \\
\hline Nurse/bed ratio & 1.16 & 1.16 & 1.16 \\
\hline \multicolumn{4}{|l|}{ Full-time equivalent positions } \\
\hline Physicians & 13 & 12 & 12 \\
\hline Registered nurses & 17.25 & 19.25 & 20.25 \\
\hline Nursing assistants & 4.95 & 3.1 & 2.1 \\
\hline Unit nurse director & 1.0 & 1.0 & 1.0 \\
\hline Clinical nurse specialist & 1.0 & 1.0 & 1.0 \\
\hline \multicolumn{4}{|l|}{ Change in positions } \\
\hline Clinical nurse specialist & - & No & No \\
\hline Unit nurse manager & - & No & Yes \\
\hline Physician leader gastrointestinal surgery & - & No & No \\
\hline Physician leader urology & - & No & Yes \\
\hline Chair of the surgical department & - & No & Yes \\
\hline \multicolumn{4}{|l|}{ Patient data and sick leave (previous 6 months) } \\
\hline Number of patient admissions per month & 192 & 174 & 173 \\
\hline Length of stay (mean days) & 3.46 & 3.63 & 3.62 \\
\hline Occupied beds & $87 \%$ & $96 \%$ & $89 \%$ \\
\hline Emergency admissions & $64 \%$ & $65 \%$ & $66 \%$ \\
\hline Sick leave nursing staff & $13.22 \%$ & $5.05 \%$ & $7.58 \%$ \\
\hline Sick leave physicians & $3.55 \%$ & $1.47 \%$ & $2.58 \%$ \\
\hline Registered adverse events by year & 2015 & 2016 & 2017 \\
\hline Numbers of reported adverse events & 38 & 42 & 52 \\
\hline
\end{tabular}

Table 2 Explanation of the selected tools and strategies implemented in study period [32]

TeamSTEPPS tools and strategies

\section{Closed-loop}

ISBAR

I-PASS

Brief

Huddle

Debrief

Task assistance

The two- challenge rule

\section{Cross monitoring}

\section{Explanation}

Using closed-loop communication to ensure that information conveyed by the sender is understood by the receiver as intended

A technique for communicating critical information that requires immediate attention and action concerning a patient's condition

Strategy designed to enhance information exchange during transitions in care

Short session prior to start to share the plan, discuss team formation, assign roles and responsibilities, establish expectations and climate, anticipate outcomes and likely contingencies

Ad hoc meeting to re-establish situational awareness, reinforce plans already in place, and assess the need to adjust the plan

Informal information exchange session designed to improve team performance and effectiveness through lessons learned and reinforcement of positive behaviors

Helping others with tasks builds a strong team. Key strategies include: Team members protect each other from work overload situations, Effective teams place all offers and requests for assistance in the context of patient safety, Team members foster a climate where it is expected that assistance will be actively sought and offered

Empowers all team members to "stop the line" if they sense or discover an essential safety breach. When an initial assertive statement is ignored: It is your responsibility to assertively voice concern at least two times to ensure that it has been heard, The team member being challenged must acknowledge that concern has been heard, If the safety issue still hasn't been addressed: Take a stronger course of action; Utilize supervisor or chain of command

A harm error reduction strategy that involves: Monitoring actions of other team members, Providing a safety net within the team, Ensuring that mistakes or oversights are caught quickly and easily, "Watching each other's back"

Tool to help assess health care delivery situations 
Table 3 Time of implementation of the selected TeamSTEPPS tools and strategies

\begin{tabular}{|c|c|c|c|c|c|c|c|c|c|}
\hline $\begin{array}{l}\text { The teamwork } \\
\text { competencies }\end{array}$ & $\begin{array}{l}\text { May } \\
2016\end{array}$ & $\begin{array}{l}\text { June } \\
2016\end{array}$ & $\begin{array}{l}\text { August } \\
2016\end{array}$ & $\begin{array}{l}\text { September } \\
2016\end{array}$ & $\begin{array}{l}\text { October } \\
2016\end{array}$ & $\begin{array}{l}\text { January } \\
2017\end{array}$ & $\begin{array}{l}\text { February } \\
2017\end{array}$ & $\begin{array}{l}\text { March } \\
2017 \\
\end{array}$ & $\begin{array}{l}\text { May } \\
2017 \\
\end{array}$ \\
\hline Communication & Closed-loop & ISBAR $^{1}$ & & & & & & & I-PASS \\
\hline Leadership & & & Briefs & Huddles & & Debriefs & & & \\
\hline $\begin{array}{l}\text { Situation } \\
\text { Monitoring }\end{array}$ & & & & & Cross monitoring & & STEP ${ }^{2}$ & & \\
\hline Mutual Support & & & & & & Task assistance & & $\begin{array}{l}\text { Two Challenge } \\
\text { rule }\end{array}$ & \\
\hline
\end{tabular}

${ }^{1}$ ISBAR = Identification, Situation, Background, Assessment, Request/Recommendation - Use by exchange of critical information

${ }^{2} \mathrm{STEP}=$ Status of the patient, Team members, Environment, Progress toward the goal - Used by focusing on updated electronic care plans

${ }^{3}$ I-PASS=Illness severity, Patient summary, Action list, Situation awareness and contingency planning, Synthesis by receiver - Systematic handoffs with focus on

patient safety risks

staff $(75 \mathrm{~min})$, and for physicians $(20 \mathrm{~min})$ were conducted 5 months after the initial team training.

After 8 months of intervention, some changes in the wards' leadership occurred. The master trained head surgeon of urology left employment at the hospital. The chair of the department moved to a higher position in the hospital organization, and the head surgeon of the gastrointestinal surgery section assumed the position of chair. The unit nurse manager was allocated to a position as assistant chair of the surgical department, and the clinical nurse specialist assumed the role of the leader of the change team (Table 1).

\section{Phase 3. Make it stick - sustainment}

Rather than reducing the intervention pressure, it was maintained, and the implementation of tools and strategies continued. Five more tools were implemented during the last 6 months of the 12-month study period (Table 3). Achievements were celebrated along the way. When conducting whiteboard patient safety huddles after rounding every day, 30 days in a row, they celebrated with a whiteboard-themed cake.

After 11 months, another refresher training session was held for the nursing staff $(75 \mathrm{~min})$, but not for the physicians (due to busy work schedules). Other than the missed refresher training, the intervention was conducted as intended, with the interprofessional change team and leadership leading the change, and with a project group that had meetings every second month throughout the project period [43].

\section{Measurements}

Three questionnaires were used to evaluate the intervention. For measuring the professional outcomes (teamwork), the TeamSTEPPS Teamwork Perceptions Questionnaire (T-TPQ) and the Collaboration and Satisfaction about Care Decisions in Teams (CSACD-T) were used, and for measuring organizational outcomes (patient safety culture), the Hospital Survey of Patient Safety Culture Questionnaire (HSOPS) was used.
The T-TPQ is a 35 -item questionnaire $[44,45]$ that measures individuals' perception of the level of teamwork that exists in their work unit. Participants responded using a 5-point Likert scale of agreement ( $5=$ strongly agree to $3=$ neutral to $1=$ strongly disagree). The T-TPQ measures five teamwork dimensions addressed in the TeamSTEPPS program; there are seven items for each of the following five dimensions: "Team structure", "Leadership", "Mutual Support", "Situational Monitoring" and "Communication".

The CSACD-T is a questionnaire measuring clinical decision making in teams. It is composed of seven items with statements regarding collaboration in team decision making about patient care and two items about satisfaction with decision making. The participants responded by using a 7-point Likert scale of agreement (from $1=$ strongly disagree to $7=$ strongly agree), global collaboration (from $1=$ no collaboration to $7=$ complete collaboration), and satisfaction about care decisions (from $1=$ not satisfied to $7=$ very satisfied). The questionnaire was developed from the original nurse-physician "Collaboration and Satisfaction about Care Decisions" questionnaire [46].

The HSOPS [47] is a questionnaire that assesses the extent to which healthcare professionals' organizational culture supports patient safety. It is recommended for evaluating the cultural impact of team training and patient safety interventions [47]. The full HSOPS comprises 2 single items and 12 patient safety culture dimensions. Each dimension is composed of three or four items [47]. The two single items ("Number of Events Reported" and "Patient Safety Grade") and two of the dimensions ("Overall Perceptions of Patient Safety" and "Frequency of Events Reported") are regarded as outcome measures. Three dimensions are regarded as hospital-level measures [48]. Because we only studied one unit, we excluded the hospital-level section of the questionnaire (11 items -3 dimensions) and used the 2 single items and the remaining 33 items of the nine unit-level dimensions: "Teamwork Within Unit", "Manager's Expectations \& Actions Promoting Patient Safety", 
"Organizational Learning - Continuous Improvement", "Feedback and Communication About Error", "Communication Openness", "Staffing", "Nonpunitive Response to Errors", "Overall Perceptions of Patient Safety", and "Frequency of Events Reported" [48]. The participants responded by using a 5 -point Likert scale of agreement (from $1=$ strongly disagree to $5=$ strongly agree, with "neither" in the middle) or frequency (from $1=$ very seldom to $5=$ very often). The single item "Patient Safety Grade", which asks participants to provide an overall grade on patient safety for their unit, has the following five response options: $\mathrm{A}=$ Excellent $\mathrm{B}=$ Very Good, $\mathrm{C}=$ Acceptable, D = Poor, E = Failing. The single item "Number of Events Reported", which indicates the number of adverse events the participants have reported over the past 12 months, has six response options: $1=$ No events, $2=1$ to 2 events, $3=3$ to 5 events, $4=6$ to 10 events, $5=11$ to 20 events, $6=21$ events or more [47].

All three questionnaires were translated into Norwegian and psychometrically tested [49-51]. In addition to the questionnaires, participants' background information was solicited (sex, age group, profession group, and employee time in the unit).

\section{Data collection}

An electronic survey (SurveyXact) was distributed by email to the healthcare professionals to evaluate the effect of the TeamSTEPPS program. Data collection was conducted at baseline (February-March 2016) and after 6 months (November-December 2016) and 12 months of intervention (June 2017). Unit profile data were collected from the unit nurse manager.

\section{Statistical analyses}

To test for statistically significant changes between baseline and 6 months and between baseline and 12 months, a paired t-test was applied on the healthcare professional's mean scores of the T-TPQ and HSOPS dimensions and the total score of the CSACD-T, and a Wilcoxon signedrank test was applied on the two single items of the HSOPS [52]. A generalized linear mixed model (GLMM) [53] was used to investigate the outcome of TeamSTEPPS by estimating the associations among the nine HSOPS dimensions used as dependent variables and "Profession group" (nursing staff and physicians) and "Time" (baseline, after 6 and 12 months of intervention) as the two independent variables. A GLMM is a generalization of traditional linear regression that adjusts for the correlation between repeated measurements within each subject and finds the best linear fit to the data across all individuals. The model maximizes power by utilizing all data despite missing observations in some subjects [54, 55]. The GLMM was applied to the total sample $(n=98)$, and the results are reported as estimates with $95 \%$ confidence intervals. To test whether any of the three significant improved teamwork dimensions of the T-TPQ were associated with two of the patient safety culture outcomes ("Overall patient safety" and "Patient Safety Grade") after 12 months of intervention, multiple linear regression analysis was performed on all healthcare professionals $(n=$ 31) who responded after 12 months of intervention [56]. A $p$-value $<.05$ was considered to be statistically significant for all analyses. Statistical Package for Social Sciences (SPSS) version 24 (Armonk, New York) and R 3.1.1 were used to analyze the data. The study adheres to the Transparent Reporting of Evaluations with Nonrandomized Designs (TREND) guidelines [57].

\section{Results}

Of the 43 invited healthcare professionals in the ward, 35 of them responded to the survey at baseline. After 6 months of the intervention, 32 healthcare professionals responded, of which 28 had also responded at baseline. After 12 months of the intervention, 31 healthcare professionals responded, of which 25 had responded at baseline. A total of 98 responses from all respondents were collected at the three time points. See Table 4 for an overview. The characteristics of the respondents are displayed in Table 5.

The mean scores on the T-TPQ, CSACD-T and HSOPS for those answered two times (baseline and after 6 months or baseline and after 12 months) are displayed in Table 6. None of the teamwork dimensions of the TTPQ showed significant changes after 6 months. After 12 months of intervention, significant improvements were found in three teamwork dimensions, regarded as professional outcomes: "Situation Monitoring", "Mutual Support", and "Communication". No significant changes were found in the professional outcome "Team decision making" (CSACD-T) during the study period.

The patient safety culture results (HSOPS), regarded as organizational outcomes, showed significantly improved scores in two dimensions after 6 months of intervention: "Organizational Learning \& Continuous Improvement" and "Communication Openness". The three dimensions "Communication Openness", "Teamwork Within Unit" and

Table 4 Samples and respondents

\begin{tabular}{llll}
\hline & Sample & $\mathbf{n}$ & Response rate \\
\hline Baseline & 43 & 35 & $81 \%$ \\
After 6 months of intervention & 42 & 32 & $76 \%$ \\
After 12 months of intervention & 40 & 31 & $78 \%$ \\
In total & & 98 & \\
Both baseline and after 6 months & & 28 & \\
Both baseline and after 12 months & & 25 & \\
\hline
\end{tabular}


Table $\mathbf{5}$ Characteristics of the respondents

\begin{tabular}{lll}
\hline & $\begin{array}{l}\boldsymbol{n}=\mathbf{2 8} \\
\mathbf{6} \text { months } \\
\mathbf{n}(\%)\end{array}$ & $\begin{array}{l}\mathbf{n}=\mathbf{2 5} \\
\mathbf{1 2} \text { months } \\
\mathbf{n}(\%)\end{array}$ \\
\hline $\begin{array}{ll}\text { Gender } \\
\text { Female }\end{array}$ & $22(82)$ \\
Male & $5(18)$ & $3(12)$ \\
Profession & & \\
Physicians & $6(21)$ & $4(16)$ \\
Assistant nurses & $4(14)$ & $3(12)$ \\
Registered nurses & $18(64)$ & $18(72)$ \\
Age & & $4(16)$ \\
$\leq 30$ years & $6(22)$ & $12(48)$ \\
31-50 years & $12(44)$ & $9(36)$ \\
$\geq 51$ years & $9(33)$ & \\
Missing & 1 & $2(8)$ \\
Time employed in the unit & & $12(50)$ \\
0-5 years & $6(25)$ & $10(42)$ \\
6-15 years & $11(46)$ & 1 \\
$\geq 16$ years & $7(29)$ & \\
Missing & 4 & \\
\hline
\end{tabular}

"Manager's Expectations \& Actions Promoting Patient Safety" were significantly improved after 12 months.

The results of the GLMM estimates of organizational outcome (patient safety culture outcome) showed that both 'Organizational Learning and Continuous Improvement' and 'Communication Openness' had a significant effect after 6 months. Overall, physicians had a significant positive, as effect compared to nursing staff, on both 'Frequency of Events Reported' and 'Patient Safety Grade" (Table 7).

The multiple linear regression analysis of all respondents after 12 months $(n=31)$ found that the three improved teamwork dimensions "Situational Monitoring", "Mutual Support" and "Communication" (independent variables) explained $31.6 \%$ of the variance in the "Patient Safety Grade" after 12 months of intervention. The model reached statistical significance $(p=.012)$. When analyzing which of the three independent variables contributed to the prediction of "Patient Safety Grade", the model showed that "Mutual Support" had the largest $\beta$ coefficient $(\beta=.76)$ and that the effect was significant $(p=.036)$. When testing with the "Overall Perceptions of Patient Safety" as the dependent variable, the model reached statistical significance $(p=.021)$. The three teamwork dimensions explained $24.3 \%$ of the variance in the "Overall Perceptions of Patient Safety" after 12 months of intervention but with a low ß-coefficient and without statistical significance.

\section{Discussion}

Regarding organizational outcomes as related to the SEIPS 2.0 model, improvements were found in two patient safety culture dimensions after the first 6 months of this comprehensive intervention. No improvement was found in professional outcome after the first 6 months, as measured by perceptions of teamwork. After the full 12 months, however, improvements were found in both professional and organizational outcomes. Improvement in professional outcomes were shown in three out of four perceptions of teamwork dimensions. Regarding organizational outcomes, improvements were found in three patient safety culture dimensions. These results indicate that the team training program had an effect after 12 months of implementation. The GLMM estimates demonstrated an effect of time on the patient safety culture dimensions (organizational outcome) "Organizational Learning and Continuous Improvement" and "Communication Openness" after 6 months, and the estimates also demonstrated that physicians had an overall positive significant effect compared to nursing staff on the patient safety culture dimensions "Frequency of Events Reported" and "Patient Safety Grade". Furthermore, the teamwork dimension "Mutual Support" was associated with "Patient Safety Grade" after 12 months of intervention.

No significant improvement after 6 months in T-TPQ measures may be explained by the fact that few of the TeamSTEPPS tools had been implemented by that point. However, we expected to find improvement in "Communication" after 6 months since the tools Closed-loop and ISBAR (Identification, Situation, Background, Assessment, Request/Recommendation) were implemented in the work system in an early phase of the intervention. After 12 months of intervention, however, the results showed improvement in three teamwork dimensions ("Situation Monitoring", "Mutual Support", and "Communication"). The cross-monitoring strategy was implemented after 5 months, and the STEP (Status of the patient, Team members, Environment, Progress toward the goal) tool was implemented after 9 months [58], so the improvement in "Situation Monitoring" may be due to the implementation of these tools. "Situation Monitoring" involves continuously scanning the environment for important information, watching out for other team members, exchanging relevant information, and jointly reevaluating patient goals [44]. The improved scores in "Mutual Support" may be a result of the "Task Assistance" and "Two Challenge Rule" strategies that were implemented in the work system during the study period [58]. "Mutual Support" is about cautioning each other about potentially risky patient safety situations and about assisting one another during high workloads [44]. When observing these improvements in teamwork 
Table 6 Healthcare professional perceptions of teamwork and patient safety culture from baseline to 6 and 12 months of intervention

\begin{tabular}{|c|c|c|c|c|c|c|c|c|}
\hline & \multicolumn{4}{|l|}{$n=28$} & \multicolumn{4}{|l|}{$n=25$} \\
\hline & \multirow[t]{2}{*}{$\begin{array}{l}\text { baseline } \\
\text { mean }\end{array}$} & \multirow[t]{2}{*}{$\begin{array}{l}6 \text { months } \\
\text { mean }\end{array}$} & \multicolumn{2}{|c|}{$\begin{array}{l}\text { change from } \\
\text { baseline } \\
\text { to } 6 \text { months }\end{array}$} & \multirow[t]{2}{*}{$\begin{array}{l}\text { baseline } \\
\text { mean }\end{array}$} & \multirow[t]{2}{*}{$\begin{array}{l}12 \\
\text { months } \\
\text { mean }\end{array}$} & \multicolumn{2}{|c|}{$\begin{array}{l}\text { change from } \\
\text { baseline } \\
\text { to } 12 \text { months }\end{array}$} \\
\hline & & & $\mathbf{t}^{1}$ & $\mathrm{p}^{1}$ & & & $\mathrm{t}^{1}$ & $\mathrm{p}^{1}$ \\
\hline \multicolumn{9}{|l|}{$\mathrm{T}-\mathrm{TPQ}^{2}$ dimensions } \\
\hline Team Function & $3.93(.40)$ & $3.96(.44)$ & .48 & .638 & $3.95(.43)$ & $4.08(.44)$ & 1.71 & .100 \\
\hline Leadership & $4.24(.40)$ & $4.21(.49)$ & -.39 & .700 & $4.16(.39)$ & $4.15(.63)$ & -.09 & .926 \\
\hline Situation Monitoring & $3.79(.47)$ & $3.98(.56)$ & 1.74 & .094 & $3.70(.43)$ & $4.06(.54)$ & 4.70 & .001 \\
\hline Mutual Support & $3.85(.44)$ & $3.93(.51)$ & .89 & .382 & $3.83(.44)$ & $4.03(.50)$ & 1.04 & .027 \\
\hline Communication & $3.84(.40)$ & $3.94(.50)$ & 3.34 & .345 & $3.81(.39)$ & $4.02(.53)$ & 2.66 & .015 \\
\hline \multicolumn{9}{|l|}{ CSACD-T ${ }^{3}$} \\
\hline Team Decision Making & $4.73(.89)$ & $5.02(1.09)$ & 1.29 & .207 & $4.69(.92)$ & $4.95(1.03)$ & 1.32 & .200 \\
\hline \multicolumn{9}{|l|}{ HSOPS $^{4}$ dimensions } \\
\hline Teamwork Within Unit & $3.87(.54)$ & $4.08(.52)$ & 1.80 & .084 & $3.78(.52)$ & $4.05(.51)$ & 2.39 & .025 \\
\hline Manager Expect. \& Actions Promoting Pat. Safety & $4.18(.60)$ & $4.29(.50)$ & .91 & .370 & $4.11(.56)$ & $4.39(.52)$ & 2.72 & .012 \\
\hline Organizational Learning - Cont. Improvement & $3.82(.51)$ & $4.05(.61)$ & 1.8 & .001 & $3.76(.51)$ & $3.97(.65)$ & 1.78 & .087 \\
\hline Feedback \& Communication About Error & $3.71(.62)$ & $3.85(.70)$ & .04 & .965 & $3.65(.58)$ & $3.90(.60)$ & 1.84 & .078 \\
\hline Communication Openness & $3.83(.49)$ & $4.07(.60)$ & 2.37 & .025 & $3.77(.59)$ & $3.97(.49)$ & 2.58 & .017 \\
\hline Staffing & $3.52(.46)$ & $3.39(.52)$ & -1.08 & .292 & $3.81(.49)$ & $4.07(.53)$ & .06 & .955 \\
\hline Nonpunitive Response to Errors & $2.90(.69)$ & $3.14(.83)$ & 1.38 & .178 & $2.86(.66)$ & $3.01(.84)$ & .97 & .342 \\
\hline Frequency of Events Reported ${ }^{5}$ & $2.88(.70)$ & $3.13(.84)$ & 1.98 & .059 & $3.49(.45)$ & $3.50(.66)$ & 1.09 & .287 \\
\hline Overall Perceptions of Patient Safety ${ }^{5}$ & $4.12(.51)$ & $4.28(.50)$ & .90 & .375 & $4.13(.49)$ & $4.27(.62)$ & 1.94 & .065 \\
\hline HSOPS $^{4}$ single items & & & $z$-score ${ }^{6}$ & $p^{6}$ & & & $z$-score ${ }^{6}$ & $p^{6}$ \\
\hline Number of Events Reported ${ }^{5}$ & $2.11(.83)$ & $2.00(.80)$ & -.63 & .527 & $2.24(.78)$ & $2.15(.72)$ & -.78 & .439 \\
\hline Patient Safety Grade 5 & $3.67(.56)$ & $3.79(.59)$ & -.82 & .414 & $3.67(.57)$ & $3.92(.56)$ & -.1 .9 & .059 \\
\hline
\end{tabular}

${ }^{1}$ Paired t-test

${ }^{2} \mathrm{~T}$-TPQ $=$ TeamSTEPPS Teamwork Perceptions Questionnaire (scale 1-5)

${ }^{3}$ CSACD-T = Collaboration and Satisfaction About Care Decisions in Teams Questionnaire (scale 1-7)

${ }^{4}$ HSOPS $=$ Hospital Survey of Patient Safety Culture Questionnaire (scale 1-5)

${ }^{5}$ Patient Safety outcome measures

${ }^{6}$ Wilcoxon Signed Ranks Test

dimensions from a system perspective, they are seen as improved professional outcomes (see Fig. 1). Previous studies from the context of surgical wards that have measured self-reported teamwork have produced ambiguous results [59-61]. Paull, DeLeeuw [61] found improvement in all scores in their multicenter study when the scores were measured immediately after the training. Study results collected a short time after a team training may benefit from the positive experience the participants have just had and can be seen to reflect a strong Hawthorne effect [62]. The reason why we did not see improvements in team decision making in our study may be due to the time points selected for measurement. Previous studies that showed enhanced scores in decision making measured 2 weeks and 2 months after simulation training $[63,64]$. Our results for team decision making may also be explained by the fact that the TeamSTEPPS program does not emphasize decision making, and therefore, there was not a focus on this important aspect of teamwork in the intervention. In the teamwork literature from Europe, where team competencies are referred to as team skills, decision making is one of the six skills in the definition of non-technical skills (NTS) [65]. Furthermore, decision making has also recently been emphasized in the teamwork literature, indicating significance for patient safety and patient outcomes $[2,34,35]$.

The organizational outcome measured by patient safety culture showed improvement in "Organizational Learning \& Continuous Improvement" and "Communication Openness" after 6 months of intervention, and improvement in the latter was sustained after 12 months, both of which are interesting results. "Communication 
Table 7 Estimated Patient Safety Culture by "Time" and "Profession group" ( $n=98)$

\begin{tabular}{|c|c|c|c|}
\hline Parameter & Estimate & 95\% Confidence Interval & $p^{1}$ \\
\hline \multicolumn{4}{|c|}{ Organizational Learning and Continuous Improvement } \\
\hline Intercept & 3.80 & $3.60,4.00$ & .000 \\
\hline Baseline $^{2}$ & $0^{b}$ & & \\
\hline 6 months of intervention & .33 & $.05,60$ & .020 \\
\hline 12 months of intervention & .18 & $-.09, .46$ & .193 \\
\hline Nursing staff ${ }^{2}$ & $0^{b}$ & & \\
\hline Physicians & -.27 & $-.54, .00$ & .051 \\
\hline \multicolumn{4}{|l|}{ Communication Openness } \\
\hline Intercept & 3.80 & $3.63,4,02$ & .000 \\
\hline Baseline $^{2}$ & $0^{\mathrm{b}}$ & & \\
\hline 6 months of intervention & .29 & $.02, .55$ & .035 \\
\hline 12 months of intervention & .21 & $-.05, .48$ & .116 \\
\hline Nursing staff ${ }^{2}$ & $0^{\mathrm{b}}$ & & \\
\hline Physicians & -.12 & $-.38, .14$ & .366 \\
\hline \multicolumn{4}{|l|}{ Frequency of Events Reported } \\
\hline Intercept & 2.73 & $2.46,3.00$ & .000 \\
\hline Baseline $^{2}$ & $0^{b}$ & & \\
\hline 6 months of intervention & .26 & $-.11, .63$ & .164 \\
\hline 12 months of intervention & .13 & $-.25, .51$ & .500 \\
\hline Nursing staff ${ }^{2}$ & $0^{b}$ & & \\
\hline Physicians & .56 & $.19, .93$ & .003 \\
\hline \multicolumn{4}{|l|}{ Patient Safety Grade } \\
\hline Intercept & 3.60 & $3.41,3.79$ & .000 \\
\hline Baseline $^{2}$ & $0^{\mathrm{b}}$ & & \\
\hline 6 months of intervention & .11 & $-.16, .38$ & .410 \\
\hline 12 months of intervention & .25 & $-.02, .52$ & .074 \\
\hline Nursing staff ${ }^{2}$ & $0^{b}$ & & \\
\hline Physicians & .40 & $.14, .66$ & .003 \\
\hline
\end{tabular}

Openness" is a measure of whether staff freely speak up if they see something that may negatively affect a patient and if they feel free to question those with more authority than themselves [66]. This result is therefore of importance regarding the patient safety culture in the ward, as it may contribute to catching adverse events before it reaches a patient. Regarding whether the healthcare professionals reported diverse types of adverse events in our study, the average answer was "sometimes" at all data collection times, while the registered adverse events increased during the study period. An increase in adverse events is not desirable, but may be seen as an improvement in the reporting culture. The main purpose of reporting is to learn from adverse events [67], and learning is an important part of the human factors approach to patient safety. After 6 months, improvements were found in organizational outcomes (in two patient safety dimensions). After the full 12 months, improvements were found in both organizational outcomes (three patient safety culture dimensions) and professional outcomes (three teamwork dimensions). The mixed model estimates demonstrated that physicians had effects on two patient safety culture measures. Furthermore, the results showed that teamwork was associated with Patient Safety Grade [68]. The improvement in the HSOPS dimension "Organizational Learning - Continuous Improvement" (organizational outcome) may indicate that the healthcare professionals perceived their ward as a learning unit. This result also supports the mixed model estimate, which demonstrated that the time had an effect on "Organizational Learning \& Continuous Improvement" after 6 months. The estimates also demonstrated that the healthcare professionals' perceptions of "Communication Openness" were affected by time (6 months), which corresponds with the results from the t-test analyses, where "Communication Openness" showed significant 
improvements after both 6 and 12 months. The estimates from the mixed models that suggested that physicians had a positive effect on the intervention compared to nursing staff on two patient safety culture dimensions is an interesting finding since it is often challenging to involve physicians in interprofessional interventions in wards [69]. Although we cannot say for sure what caused what, we consider the interprofessional approach to training and implementation as crucial to success in the quality improvement of teamwork and patient safety work in hospital wards. The interprofessional approach may have influenced the professional and organizational outcomes in a positive way. In addition to the sustained improvement in "Communication Openness", two more dimensions of HSOPS were improved after 12 months: "Teamwork Within Unit" and "Manager's Expectations \& Actions Promoting Patient Safety". As a part of an enabling work environment, management and leadership are important enablers in achieving effective teamwork and patient safety in complex organizations [70]. This teamwork and patient safety intervention, led by the leaders and the other members of the change team, may have contributed to improvements in these dimensions. The changes in leadership positions may also have accounted for the improvement, but this is uncertain. However, although the master trained nurse unit manager resigned from the unit, she continued to work in the administration of the department and continued to give support and guidance for the intervention from her new position.

Our improved patient safety culture results in three dimensions of the HSOPS (organizational outcome) are in line with those from previous research in diverse hospital contexts. Two multicenter studies found improvement in three HSOPS dimensions when measured after 12 months [71, 72], and Thomas and Galla [69] found improvements in three HSOPS dimensions after 2 years. Schwartz, Welsh [72] found a decrease from 6 to 12 months in their multicenter study, a decrease they explained with a need for early refresher training.

The improved professional outcome "Mutual Support" was associated with "Patient Safety Grade" at the end of the study period, which is interesting from a human factors perspective since this T-TPQ dimension Mutual Support encompasses items focus on patient safety and emphasizes the strong patient safety aspect of the TeamSTEPPS program.

The use of the conceptual framework contributed to an enhanced understanding of the system approach in our study, which is important to implement and sustain innovations [73]. When implementing teamwork tools, such as ISBAR, Closed-loop, and Cross-monitoring [58] in the work system, the use of the tools and strategies in the clinical work processes have influenced professional outcomes indicating that the teamwork competencies of the healthcare professionals improved during the study period. Transfer of the learning from team training is crucial to patient safety and interesting from a human factors perspective, as outcomes are influenced by the learning-to-transfer pathway [74]. The improvement in organizational outcomes (patient safety culture) may be due to the TeamSTEPPS intervention in the work system (see Fig. 1).

The implementation of teamwork tools that initiated new ways of working may in time lead to system changes, but that was beyond the scope of this study. The healthcare professionals in hospital wards are organized in silos and system changes and structural changes that promote teamwork and patient safety are warranted in the future [1].

\section{Study limitations}

The study has some limitations. The lack of randomization and controls may have threatened the internal validity, although a pre-post design is useful where there are practical barriers to a randomized design [75]. The study samples were small, but the response rates were satisfying, without risk of response bias. Because of the uncontrolled design, we cannot conclude that the improvements were due to the intervention. There are always secular trends that might be occurring at the same time in a surgical ward, and which may have influenced our results [76]. However, because of these study limitations, caution must be taken in generalizing the results.

\section{Conclusions}

This study showed the effect of a human factors team training intervention after 12 months of implementation in a surgical ward, an effect that was demonstrated by both professional and organizational outcomes in the SEIPS 2.0 model. More work needs to be done to investigate the effect of TeamSTEPPS interventions in surgical wards, and studies with larger sample sizes and stronger designs are preferred. Future studies testing the causal pathways identified by SEIPS 2.0 will be of special interest.

\section{Abbreviations \\ CSACD-T: Collaboration and Satisfaction with Care Decisions in Teams; GLMM: Generalized Linear Mixed Model; HSOPS: The Hospital Survey of Patient Safety Culture; ISBAR: Identification, Situation, Background, Assessment, Request/Recommendation; SEIPS: Systems Engineering Initiative for Patient Safety; STEP: Status of the patient, Team members, Environment, Progress toward the goal; I-PASS: Illness severity, Patient summary, Action list, Situation awareness and contingency planning, Synthesis by receiver; TeamSTEPPS: Team Strategies and Tools to Enhance Performance and Patient Safety; T-TPQ: TeamSTEPPS Teamwork Perceptions Questionnaire}

\section{Acknowledgments}

We would like to thank the healthcare professionals from the surgical ward who participated in the study. We also wish to thank associate professor Randi Tosterud (NTNU) and senior consultant Terje Ødegården, Center for Simulation and Patient Safety (NTNU), for their valuable facilitation of the simulation training. Last, we want to thank Principal Statistician Tristan R. 
Grogan, University of California, Los Angeles (UCLA), and Professor Jo Røislien at the University of Stavanger (UIS), for consulting on the data analysis.

\section{Authors' contributions}

ORA, MLHL, SEH, and RB contributed to the conception and design and the writing and critical revision of the manuscript and approved the final version published.

\section{Funding}

This work was supported by the Norwegian Nurses Organization (15/0018). The Norwegian Nurses Organization had no role in the study design, collection, analysis and interpretation of the data or in the writing of the manuscript.

\section{Availability of data and materials}

The datasets used during the current study are available from the corresponding author on reasonable request.

\section{Ethics approval and consent to participate}

The study protocol was reviewed by the "Regional Committees for Medical Research Ethics - South East Norway" (ref. 2016/1013 C). The study was approved by the Norwegian Center for Research Data (ref. no. 46323), and conducted in accordance with the Helsinki Declaration [77]. Written information about the study was sent to all participants via SurveyXact with reference to the principle of autonomy addressed by confidentiality and voluntariness. Although the team training and implementation activities were compulsory during work hours, participating in the surveys was voluntary. Completion of the surveys was regarded as informed consent. For ethical reasons, we did not collect data about the non-responders.

\section{Consent for publication}

"Not applicable".

\section{Competing interests}

The authors declare that they have no competing interests.

\section{Author details}

'Department of Health and Nursing Science, Faculty of Health and Sport Sciences, University of Agder, Universitetsveien 25 A, 4630 Kristiansand, Norge. ${ }^{2}$ Department of Health Science, Faculty of Medicine and Health Sciences, Norwegian University of Science and Technology, Teknologivegen 22, 2815 Gjøvik, Norway. ${ }^{3}$ Department of Quality and Health Technology, Faculty of Health Sciences, University of Stavanger, Kjell Arholmsgate 41, 4036 Stavanger, Norway. ${ }^{4}$ Department of Health Sciences, Faculty of Health Science and Technology, Karlstad University, Universitetsgatan 2, 65188 Karlstad, Sweden. ${ }^{5}$ Research Group of Nursing and Health Care Sciences, Stavanger University Hospital, Gerd-Ragna Bloch Thorsens gate 8, 4011 Stavanger, Norway.

Received: 10 April 2020 Accepted: 9 January 2021

Published online: 03 February 2021

\section{References}

1. Leape LL. Patient safety in the era of healthcare reform. Clin Orthop Relat Res. 2015:473(5):1568-73.

2. WHO. 10 facts on patient safety. 2018; Available from: https://www.who.int/ features/factfiles/patient_safety/en/. [cited 2019 April 18]

3. de Vries EN, et al. The incidence and nature of in-hospital adverse events: a systematic review. Qual Saf Health Care. 2008;17(3):216-23.

4. de Vries EN, et al. Effect of a comprehensive surgical safety system on patient outcomes. N Engl J Med. 2010;363(20):1928-37.

5. Carayon P, et al. Safety by design - work system design for patient safety: the SEIPS model. Qual Saf Health Care. 2006;15:50-8.

6. The Joint Commission. Sentinel event statistics released for 2014. [report] 2015; Available from: http://www.jointcommission.org/. [cited 2015 101015].

7. WHO. Patient safety. 2015 01.12.2015]; Available from: http://www.euro.who. int/en/health-topics/Health-systems/patient-safety/patient-safety.

8. Joint Commission, Patient Safety Systems (PS) CAMH Update 2, in The accreditation program's Comprehensive Accreditation Manual. 2016.
9. Carayon P, Wood KE. Patient safety - the role of human factors and systems engineering. Stud Health Technol Inform. 2010;153:23-46.

10. Kannampallil TG, et al. Considering complexity in healthcare systems. J Biomed Inform. 2011:44(6):943-7.

11. Marshall DC, Finlayson MP. Identifying the nontechnical skills required of nurses in general surgical wards. J Clin Nurs. 2018;27(7-8):1475-87.

12. Mache $S$, et al. General and visceral surgery practice in German hospitals: a real-time work analysis on surgeons' work flow. 2010;395(1):81.

13. National Center for Human Factors in Healthcare. 2020; Available from: https://www.medicalhumanfactors.net/. [cited 2020 January 23]

14. Holden RJ, Cornet VP, Valdez RS. Patient ergonomics: 10-year mapping review of patient-centered human factors. Appl Ergon. 2020;82:102972.

15. Russ $A L$, et al. The science of human factors: separating fact from fiction. BMJ Qual Saf. 2013;22(10):802-8.

16. Carayon $\mathrm{P}$, et al. Challenges and opportunities for improving Patient Safety through human factors and systems engineering. Health Aff. 2018:37(11):1862-9.

17. Carayon P. Human factors in patient safety as an innovation. Appl Ergon. 2010;41(5):657-65.

18. WHO. Human factors in patient safety: review of topics and tools. [Report] 2009; Available from: http://testing.chfg.org/resources/10_qrt01/WHO_PS_ HF_Review.pdf. [cited 2015 March 15]

19. Cannon-Bowers JA, et al. In: Guzzo RA, Salas E, editors. Defining Competencies and Establishing Team Training Requirements, in Team Effectiveness and Decision Making in Organizations. San Francisco: JosseyBBass; 1995. p. 333-80

20. Baker DP, et al. The Relation between Teamwork and Patient Safety, in Handbook of Human Factors and Ergonomics in Health Care and Patient Safety. 2nd ed. Boca Raton: CRC Press; 2012. p. 185-98.

21. Weaver SJ, Dy SM, Rosen MA. Team-training in healthcare: a narrative synthesis of the literature. BMJ Qual Saf. 2014;23(5):359-72.

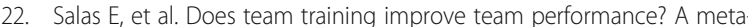
analysis. Hum Factors. 2008;50(6):903-33.

23. Weaver SJ, et al. Promoting a culture of safety as a patient safety strategy: a systematic review. Ann Intern Med. 2013;158(5 Pt 2):369-74.

24. Sacks GD, et al. Teamwork, communication and safety climate: a systematic review of interventions to improve surgical culture. BMJ Qual Saf. 2015;24(7): 458-67.

25. Howell A-M, et al. Reducing the burden of surgical harm: a systematic review of the interventions used to reduce adverse events in surgery. Ann Surg. 2014;259(4):630-41

26. Neily J, et al. Association between implementation of a medical team training program and surgical mortality. Jama. 2010;304(15):1693-700.

27. Aaberg OR, Wiig S. Interprofessional team training in hospital wards: A literature review. In European Safety and Reliability Conference (ESREL). 2017. Portoroz, Slovenia: CRC Press; 2017.

28. Rosen MA, et al. Teamwork in healthcare: key discoveries enabling safer, high-quality care. Am Psychol. 2018;73(4):433.

29. Korner $M$, et al. Relationship of organizational culture, teamwork and job satisfaction in interprofessional teams. BMC Health Serv Res. 2015;15:243.

30. El-Jardali $F$, et al. Predictors and outcomes of patient safety culture in hospitals. BMC Health Serv Res. 2011;11(1):45.

31. King HB, TeamSTEPPS, et al. Team Strategies and Tools to Enhance Performance and Patient Safety. In: Henriksen K, et al., editors. Advances in Patient Safety: New Directions and Alternative Approaches (Vol. 3: Performance and Tools). Rockville: Agency for Healthcare Research and Quality (US); 2008.

32. TeamSTEPPS 2.0. 2014 June 2019; Available from: https://www.ahrq.gov/ teamstepps/instructor/index.html. [cited 2019 August 12]

33. Stewart GL, Manges KA, Ward MM. Empowering sustained Patient Safety: the benefits of combining top-down and bottom-up approaches. J Nurs Care Qual. 2015;30(3):240-6.

34. Reader TW, Team Decision Making. In: Salas E, Rico R, Passmore J, editors. The Wiley Blackwell Handbook of the Psychology of Team Working and Collaborative Processes. West Sussex: Black Wiley; 2017. p. 271-96.

35. Salas E, Cannon-Bowers JA, Johnston JH. In: Zsambok C, Klein G, editors. How can you turn a team of experts into an expert team? Emerging training strategies, in Naturalistic Decision Making. New York: Psychology Press; 2014. p. 359-70.

36. Zavala $A M$, et al. Decision-making under pressure: medical errors in uncertain and dynamic environments. Aust Health Rev. 2018:42(4): 395-402. 
37. Dekeyser Ganz F, et al. Development of a model of Interprofessional shared clinical decision making in the ICU: a mixed-methods study. Crit Care Med. 2016:44(4):680-9.

38. Carayon $\mathrm{P}$, et al. Human factors systems approach to healthcare quality and patient safety. Appl Ergon. 2014;45(1):14-25.

39. Holden RJ, et al. SEIPS 2.0: a human factors framework for studying and improving the work of healthcare professionals and patients. Ergonomics. 2013;56(11):1669-86.

40. Ballangrud R, et al. "teamwork in hospitals": a quasi-experimental study protocol applying a human factors approach. BMC Nurs. 2017;16:34.

41. Clinical Human Factors Group. Implementing Human Factors in Healthcare 'Taking further steps'. 'How to' Guide to Human Factors 2013; Available from: http://www.improvementacademy.org/documents/Projects/human factors/Implementing-human-factors-in-healthcare-How-to-guide-volume-2FINAL-2013 05 16.pdf. [cited 2019 May 07 2019]

42. TeamSTEPPS 2.0. Course Evaluation. 2014 Content last reviewed March 2014; Available from: https://www.ahrq.gov/teamstepps/instructor/ reference/smpcefm.html. [cited 2019 February 24]

43. Davidson KW, et al. Evidence-based behavioral medicine: what is it and how do we achieve it? Ann Behav Med. 2003;26(3):161-71.

44. American Institutes for Research. TeamSTEPPS ${ }^{\oplus}$ Teamwork Perceptions Questionnaire Manual. 2010.

45. Keebler JR, et al. Validation of a teamwork perceptions measure to increase patient safety. BMJ Qual Saf. 2014;23(9):718-26.

46. Baggs JG. Development of an instrument to measure collaboration and satisfaction about care decisions. J Adv Nurs. 1994;20(1):176-82.

47. Sorra J, Dyer N. Multilevel psychometric properties of the AHRQ hospital survey on patient safety culture. BMC Health Serv Res. 2010;10:199.

48. Jones KJ, et al. In: Henriksen K, et al., editors. The AHRQ Hospital Survey on Patient Safety Culture: A Tool to Plan and Evaluate Patient Safety Programs, in Advances in Patient Safety: New Directions and Alternative Approaches (Vol. 2: Culture and Redesign). Rockville: Agency for Healthcare Research and Quality (US); 2008

49. Ballangrud R, Husebø SE, Hall-Lord ML. Cross-cultural validation and psychometric testing of the Norwegian version of the TeamSTEPPS ${ }^{*}$ teamwork perceptions questionnaire. BMC Health Serv Res. 2017;17(1):799, pp 1-10 (open access). https://doi.org/10.1186/s12913-017-2733-y.

50. Aaberg OR, et al. Collaboration and satisfaction about care decisions in team questionnaire-psychometric testing of the Norwegian version, and hospital healthcare personnel perceptions across hospital units. Nursing Open. 2019;6(2):642-50.

51. Olsen E. In: Øvretveit J, Sousa P, editors. Reliability and validity of the Hospital Survey on Patient Safety Culture at a Norwegian hospital., in Quality and safety Improvement Research: Methods and research practice from the International Quality Improvement Research Network. Lisbon, Portugal: National Scool of Public Health; 2008. p. 173-86.

52. Derrick $B$, White $P$. Comparing two samples from an individual Likert question. Int J Math Stat. 2017:18(3)

53. Bolker BM, et al. Generalized linear mixed models: a practical guide for ecology and evolution. Trends Ecol Evol. 2009;24(3):127-35.

54. West $P$, et al. Surgical programs in the veterans health administration maintain briefing and debriefing following medical team training. Jt Comm J Qual Patient Saf. 2014:40(5):235-9.

55. Katz MH. Multivariable analysis: a practical guide for clinicians and public health researchers. Cambridge: Cambridge University Press; 2011.

56. Polit, D.F. and C.T. Beck, Nursing research. Generating and assessing evidence for nursing practice. 2017, Philadelphia: Wolters Kluwer.

57. Des Jarlais DC, et al. Improving the reporting quality of nonrandomized evaluations of behavioral and public health interventions: the TREND statement. Am J Public Health. 2004;94(3):361-6.

58. Aaberg OR, et al. A complex teamwork intervention in a surgical ward in Norway. BMC Res Notes. 2019;12(1):582.

59. Kim LY. The Effects of Simulation-based TeamSTEPPS Interprofessional Communication and Teamwork Training on Patient and Provider Outcomes. UCLA. 2014

60. Riggall VK, Smith CM. Creating a sustainable, interprofessional-team training program: initial results. Clin Nurse Spec. 2015;29(3):147-55.

61. Paull DE, et al. The effect of simulation-based crew resource management training on measurable teamwork and communication among Interprofessional teams caring for postoperative patients. J Contin Educ Nurs. 2013:44(11):516-24
62. McCambridge J, Kypri K, Elbourne D. Research participation effects: a skeleton in the methodological cupboard. J Clin Epidemiol. 2014:67(8):845-9.

63. Maxson PM, et al. Enhancing nurse and physician collaboration in clinical decision making through high-fidelity interdisciplinary simulation training. Mayo Clin Proc. 2011;86(1):31-6.

64. Klipfel JM, et al. Using high-fidelity simulation to develop nurse-physician teams. J Contin Educ Nurs. 2011:42(8):347-57 quiz 358-9.

65. Flin R, Maran N. Basic concepts for crew resource management and nontechnical skills. Best Pract Res Clin Anaesthesiol. 2015:29(1):27-39.

66. Sorra J, et al. AHRQ Hospital Survey on Patient Safety Culture: User's Guide. Prepared by Westat, under Contract No. HHSA290201300003C. AHRQ Publication No. 15-0049-EF (Replaces 04-0041). Rockville: Agency for Healthcare Research and Quality, USA; 2016.

67. Weinzimmer, L.G. and C.a.J.T.J.o.a.B.S. Esken, Learning from mistakes: How mistake tolerance positively affects organizational learning and performance 2017. 53(3): p. 322-348.

68. Itoh K, Andersen HB, Madsen MD. In: Carayon P, editor. Safety Culture in Health Care, in Handbook of Human FActors and Ergonomy in Health Care and Patient Safety, vol. 823. Florida USA: CRC press: Boca Raton; 2012.

69. Thomas L, Galla C. Building a culture of safety through team training and engagement. BMJ Qual Saf. 2013;22(5):425-34.

70. Cunningham $U$, et al. Team interventions in acute hospital contexts: a systematic search of the literature using realist synthesis. BMC Health Serv Res. 2018;18(1):536.

71. Jones KJ, et al. A theory-driven, longitudinal evaluation of the impact of team training on safety culture in 24 hospitals. BMJ Qual Saf. 2013:22(5): 394-404.

72. Schwartz ME, et al. The effects of crew resource management on teamwork and safety climate at veterans health administration facilities. J Healthc Risk Manag. 2018;38(1):17-37.

73. Eccles $\mathrm{M}$, et al. Changing the behavior of healthcare professionals: the use of theory in promoting the uptake of research findings. J Clin Epidemiol. 2005:58(2):107-12

74. Salas E, et al. In: Carayon P, editor. Team Training for Patient Safety, in Human Factors and Ergonomics in Health Care and Patient Safety. Boca Raton: CRC Press; 2012.

75. Eccles $M$, et al. Research designs for studies evaluating the effectiveness of change and improvement strategies. Qual Saf Health Care. 2003;12(1):47-52.

76. Portela MC, et al. How to study improvement interventions: a brief overview of possible study types. Postgrad Med J. 2015;91(1076):343-54.

77. The World Medical Association. Declaration of Helsinki - Ethical Principles for Medical Research Involving Human Subjects. 2013; Available from: https://www.wma.net/policies-post/wma-declaration-ofhelsinki-ethical-principles-for-medical-research-involving-human-subjects/. [cited 201915 May]

\section{Publisher's Note}

Springer Nature remains neutral with regard to jurisdictional claims in published maps and institutional affiliations.
Ready to submit your research? Choose BMC and benefit from:

- fast, convenient online submission

- thorough peer review by experienced researchers in your field

- rapid publication on acceptance

- support for research data, including large and complex data types

- gold Open Access which fosters wider collaboration and increased citations

- maximum visibility for your research: over $100 \mathrm{M}$ website views per year

At BMC, research is always in progress.

Learn more biomedcentral.com/submission 